\title{
The Maximal Wrinkle Angle During the Bubble Collapse and Its Application to the Bubble Electrospinning
}

\author{
Ji-Huan $\mathrm{He}^{1,2 *}$, Man-Yu Qian ${ }^{1}$ and $Y a L^{3 *}$ \\ ${ }^{1}$ National Engineering Laboratory for Modern Silk, College of Textile and Clothing Engineering, Soochow University, Suzhou, \\ China, ${ }^{2}$ School of Mathematics and Information Science, Henan Polytechnic University, Jiaozuo, China, ${ }^{3}$ Zhejiang Sci-Tech \\ University, College of Textile Science and Engineering (International Silk College), Hangzhou, China
}

Polymer bubbles are ubiquitously used for the fabrication of nanofibers by the bubble electrospinning. When a bubble is broken, the fragments tend to be wrinkled. The wrinkle angle plays an important in controlling the fiber morphology during the bubble electrospinning. This paper shows the maximal angle is about $49^{\circ}$, which is close to the experimental value of $50^{\circ}$. This maximal angle can be used for the optimal design of the nozzle in the bubble electrospinning for the fabrication of non-smooth nanofibers.

Keywords: bubble, electrospinng, nanofiber, wrinkle, instability

\section{OPEN ACCESS}

Edited by:

Arjun Prasad Tiwari,

Jeonbuk National University, South

Korea

Reviewed by:

Xin Jing,

Hunan University of Technology,

China

*Correspondence:

Ji-Huan He

hejihuan@suda.edu.cn

Ya Li

liya@zstu.edu.cn

Specialty section:

This article was submitted to

Biomaterials,

a section of the journal

Frontiers in Materials

Received: 23 October 2021 Accepted: 21 December 2021

Published: 03 February 2022

Citation:

He J-H, Qian M-Y and Li Y (2022) The

Maximal Wrinkle Angle During the Bubble Collapse and Its Application to

the Bubble Electrospinning.

Front. Mater. 8:800567.

doi: $10.3389 /$ fmats.2021.800567

\section{INTRODUCTION}

Bubble dynamics is an old discipline, and the Young-Laplace equation (Liu and Dou, 2013) is widely used for static analysis. The bubble's rupture affects many materials' fabricating processes (Zhang et al., 2021), especially for nanofiber fabrication by bubble electrospinning (Kong, 2015; Liu et al., 2020a; Li and He, 2020; Yin et al., 2020). When a bubble is broken, many jets are ejected, or many daughter bubbles are formed (Bird et al., 2010). Oratis et al. (2020) found the wrinkle mechanism of a liquid sheet during a bubble collapse, which has attracted skyrocketing attention from various fields, including mathematics and material science. Although this mechanism is new and has great promise, a mathematical model lacks for the prediction of the wrinkle angle. Here we establish a simple formula to study the wrinkle angle.

\section{MAXIMAL WRINKLE ANGLE}

The bubble collapse and the wrinkle of the liquid sheet (Oratis et al., 2020) play an important role in the bubble electrospinning (Yin et al., 2020), Gratis et al. obtained an unparalleled achievement in the bubble collapse dynamics and found the surface tension drives the collapse and initiates its wrinkle (Oratis et al., 2020).

The surface tension depends upon the liquid sheet's curvature radius, we assume that the initial surface tension is $F_{0}$, and the initial angle between the free sheet section and the solid surface is $\alpha_{0}$, see Figure 1. During the collapse process, the liquid sheet tends to zero when it is completely collapsed, and we assume the surface tension becomes zero at its final collapse. Accordingly, we assume that the surface tension can be expressed as

$$
F(\alpha)=\frac{F_{0}}{\alpha_{0}} \alpha
$$



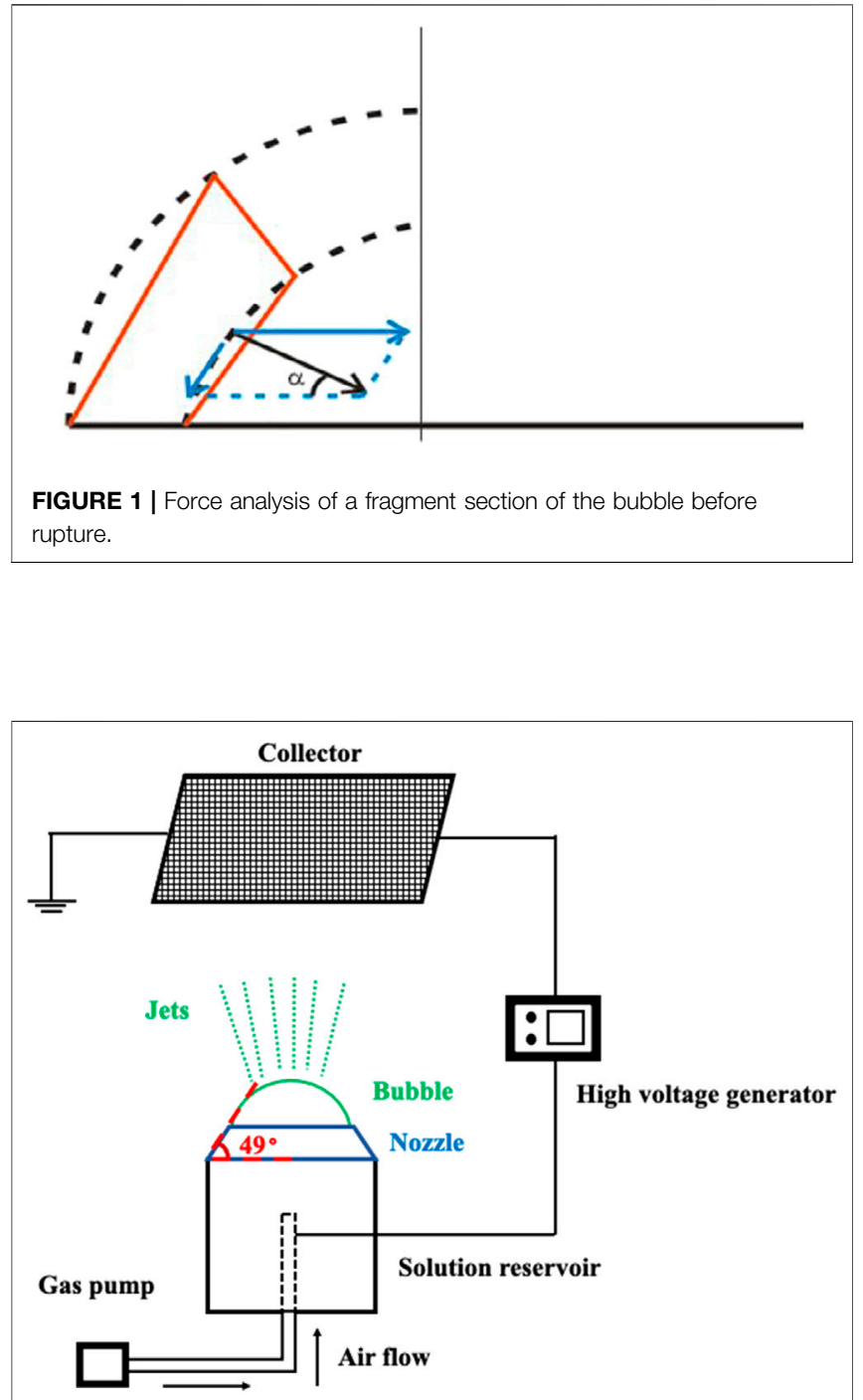

FIGURE 2 | Schematic illustration of the bubble eletrospinning.

From Eq. 1, it is obvious that the surface tension vanishes completely when the bubble is completely collapsed when $\alpha=0^{\circ}$.

The force given in Eq. 1 can be decomposed into the radial and tangential forces, as shown in Figure 1, the former is the main force for collapse, and the latter is perpendicular to the section of the sheet, and it is the main force for liquid sheet's instability, which leads finally to the wrinkle. According to Figure 1, the tangential force, $\sigma$, can be expressed as

$$
\sigma=F(\alpha) \cos \alpha=\frac{F_{0}}{\alpha_{0}} \alpha \cos \alpha
$$

The maximal $\sigma$ happens when

$$
\frac{d}{d \alpha} \sigma=\frac{F_{0}}{\alpha_{0}}(\cos \alpha-\alpha \sin \alpha)=0
$$

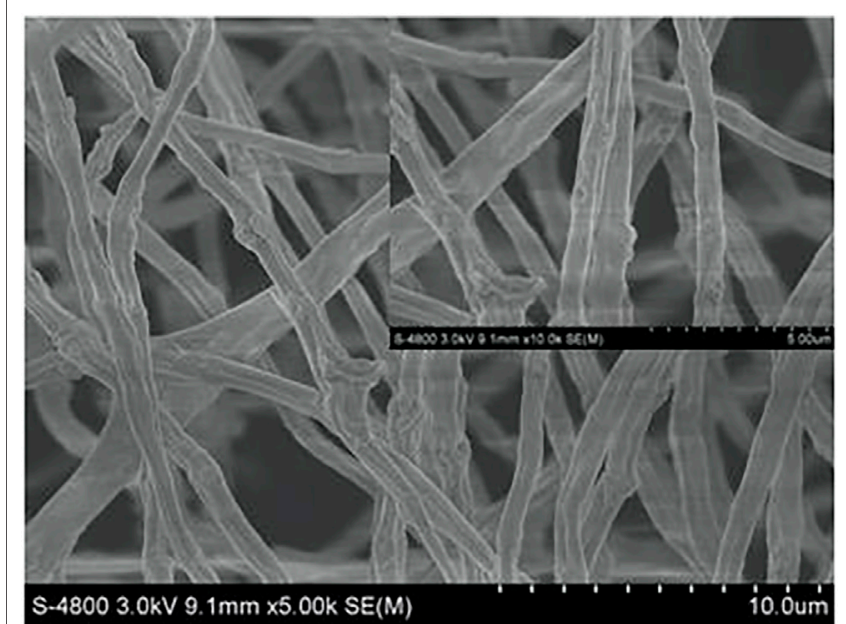

FIGURE 3 | Unsmooth fibers by controlling the bubble shape.

That is

$$
\cos \alpha-\alpha \sin \alpha=0
$$

This equation can be solved by the ancient Chinese algorithm (He, 2016). We choose two angles $\alpha_{1}=\pi / 3$ and $\alpha_{2}=\pi / 4$, and the following two residuals are obtained:

$$
R_{1}=\cos \alpha_{1}-\alpha_{1} \sin \alpha_{1}=\frac{1}{2}-\frac{\pi}{3} \times \frac{\sqrt{3}}{2}=-0.4069
$$

and

$$
R_{2}=\cos \alpha_{2}-\alpha_{2} \sin \alpha_{2}=\frac{\sqrt{2}}{2}-\frac{\pi}{4} \times \frac{\sqrt{2}}{2}=0.1517
$$

By the ancient Chinese algorithm (He, 2016), we have

$$
\alpha=\frac{R_{1} \alpha_{2}-R_{2} \alpha_{1}}{R_{1}-R_{2}}=\frac{-0.4069 \times \frac{\pi}{4}-0.1517 \times \frac{\pi}{3}}{-0.4069-0.1517}=0.2726 \pi
$$

The exact root of Eq. 4 is $\alpha=0.2737 \pi$, the relative error is 0.4\%. The ancient Chinese algorithm given in Eq. 7 was further developed into an iteration method, which was called Chun-Hui He iteration method (Khan, 2021).

When $\alpha=0.2737 \pi$ or $49^{\circ}, \sigma$ reaches its maximum; this maximal surface tension leads to instability of the fragments; as a result, the wrinkle occurs. The instability can be analyzed in a similar way as discussed in Refs. (He et al., 2021; Zuo and Liu, 2021).

\section{EXPERIMENT}

Bubble electrospinning was originally designed for the fabrication of smooth fibers (Liu et al., 2020b; Wan, 2020). In order to verify the theoretical prediction, we carried out an experiment where 
the angle between the solution surface and the bubble's wall is about $49^{\circ}$, see Figure 2 .

The spun solution was prepared by adding certain amounts of Lithium chloride ( $\mathrm{LiCl})$ dropwise to a $15 \mathrm{wt} \%$ Polyacrylonitrile/Polyethersulfone (PAN/PES) solution in $\mathrm{N}$, N-Dimethylacetamide (DMAC) following with ultrasonic excitation agitating. The weight ratio of PAN and PES was controlled at $3 / 2$, and the weight percentage of $\mathrm{LiCl}$ in the mixed solution was $1 \mathrm{wt} \%$. Afterward, the PAN/PES/LiCl blend nanofibers were obtained using a high DC voltage power supply at a $20 \mathrm{kV}$ potential and the collector with a distance of $15 \mathrm{~cm}$ from the bubble top. The SEM image of $\mathrm{PAN} / \mathrm{PES} / \mathrm{LiCl}$ nanofibers was presented in Figure 3. It is seen that we obtain the non-smooth fibers.

\section{DISCUSSION AND CONCLUSION}

This short paper gives a simple mathematical analysis, showing that the maximal wrinkle angle is about $49^{\circ}$, which is much closed to Oratis, et al.'s experimental value, which was about $50^{\circ}$. This

\section{REFERENCES}

Bird, J. C., de Ruiter, R., Courbin, L., and Stone, H. A. (2010). Daughter Bubble Cascades Produced by Folding of Ruptured Thin Films, Nature, 465, 759-762. doi:10.1038/nature09069

He, C.-H., Tian, D., Moatimid, G. M., Salman, H. F., and Zekry, M. H. (2021). Hybrid Rayleigh-van der pol-Duffing Oscillator: Stability analysis and controller. J. Low Freq. Noise, Vib. Active Control., 146134842110264. doi:10.1177/14613484211026407

He, C.-H. (2016). An Introduction to an Ancient Chinese Algorithm and its Modification. Int. J. Numer. Method. H. 26 (8), 2486-2491. doi:10.1108/hff-092015-0377

Khan, W. A. (2021). Numerical Simulation of Chun-Hui He's Iteration Method with Applications in Engineering. Int. J. Numer. Method. H. ahead-of-print. doi:10.1108/HFF-04-2021-0245

Kong, H. Y. (2015). Bubble Electrospinning and Nanofiber's Morphology Control and Applications. Soochow University. $\mathrm{PhD}$ thesis.

Li, X.-X., and He, J.-H. (2020). Bubble Electrospinning with an Auxiliary Electrode and an Auxiliary Air Flow, Nanotec, 14 (1), 42-45. doi:10.2174/ 1872210513666191107122528

Liu, F.-J., and Dou, H. (2013). A Modified Yang-Laplace Equation for the Bubble Electrospinning Considering the Effect of Humidity. Therm. Sci. 17 (2), 629-630. doi:10.2298/tsci1212290281

Liu, G.-L., Zhang, Y.-M., Tian, D., Zhou, B.-Z., Lu, Z.-Q., and Wang, C.-X. (2020). Last Patents on Bubble Electrospinning. Nanotec 14 (1), 5-9. doi:10.2174/ 1872210513666191107123446

Liu, G.-L., Zhang, Y.-M., Tian, D., Zhou, B.-Z., Lu, Z.-Q., and Wang, C.-X. (2020). Last Patents on Bubble Electrospinning. Nanotec 14 (1), 5-9. doi:10.2174/ 1872210513666191107123446

Oratis, A. T., Bush, J. W. M., Stone, H. A., and Bird, J. C. (2020). A New Wrinkle on Liquid Sheets: Turning the Mechanism of Viscous angle can be used to design the nozzle angle in the bubble electrospinning; when wrinkled nanofibers are to be fabricated, the nozzle angle should be $49^{\circ}$. If smooth nanofibers are wanted, the nozzle angle should deviate from $49^{\circ}$.

\section{AUTHOR CONTRIBUTIONS}

All authors listed have made a substantial, direct, and intellectual contribution to the work and approved it for publication.

\section{FUNDING}

The work was supported by Zhejiang Provincial Natural Science Foundation of China under Grant Nos. LQ21E030016, China Postdoctoral Science Foundation under Grant Nos. 2021M692866 and Program of Zhejiang Sci-Tech University (Grant Nos. 11110231281803). This research was also supported by "the Fundamental Research Funds of Zhejiang Sci-Tech University” (No. 2020Q002).

Bubble Collapse Upside Down. science 369, 685-688. doi:10.1126/ science.aba0593

Wan, L. Y. (2020). Bubble Electrospinning and Bubble-Spun Nanofibers. Nanotec 14 (1), 10-13. doi:10.2174/1872210513666191007114022

Yin, J., Wang, Y., and Xu, L. (2020). Numerical Approach to High-Throughput of Nanofibers by a Modified Bubble-Electrospinning. Therm. Sci. 24 (4), 2367-2375. doi:10.2298/tsci2004367y

Zhang, Z. Y., Qiang, J., Wang, S. S., Xu, M., Gan, M., Rao, Z., et al. (2021). Visualization of Bubble Nucleation and Growth Confined in 2D Flakes. Small 17 (39), e2103301, 2021 . Article 2103301. doi:10.1002/ smll.202103301

Zuo, Y., and Liu, H. (2021). Instability of the Printing Jet during the ThreeDimensional-Printing Process. J. Low Freq. Noise, Vib. Active Control. 40, 1795-1803. doi: $10.1177 / 14613484211021518$

Conflict of Interest: The authors declare that the research was conducted in the absence of any commercial or financial relationships that could be construed as a potential conflict of interest.

Publisher's Note: All claims expressed in this article are solely those of the authors and do not necessarily represent those of their affiliated organizations, or those of the publisher, the editors and the reviewers. Any product that may be evaluated in this article, or claim that may be made by its manufacturer, is not guaranteed or endorsed by the publisher.

Copyright (c) $2022 \mathrm{He}$, Qian and Li. This is an open-access article distributed under the terms of the Creative Commons Attribution License (CC BY). The use, distribution or reproduction in other forums is permitted, provided the original author(s) and the copyright owner(s) are credited and that the original publication in this journal is cited, in accordance with accepted academic practice. No use, distribution or reproduction is permitted which does not comply with these terms. 